\title{
Experiments and design of an inference fuzzy system
}

\author{
F. Benmakrouha ${ }^{1}$, C. Hespel ${ }^{1}$ E. Monnier ${ }^{1}$ and D. Quichaud ${ }^{1}$ \\ ${ }^{1}$ Computer Sciences INSA RENNES, FRANCE \\ \{benma, hespel, monnier, quichaud $\} @ i n s a-r e n n e s . f r$
}

Keywords: fuzzy system; diabetes; datum plane covering.

\begin{abstract}
The aim of this paper is to propose a criterion to estimate the design, from experimental data, of a fuzzy inference system, when data are sparse.

This lack of data is important and may improve the generalisation ability of fuzzy systems (0). Several methods have been proposed to obtain automatic fuzzy rules from sparse training data. In (0), the authors first construct fuzzy rules from collect data. Then, they use kernel regressions for generate training data.

Another technique used when classical inference methods produce sparse fuzzy rules is a diffusion procedure based on interpolation to initialize incomplete rules (0), (0), (0).

Our method has the advantage of occuring before initialization step and therefore avoiding unfired rules which make difficult to produce an accurate output. .
\end{abstract}

\section{INTRODUCTION}

The lack of data is important and is pointed out by M Lutaud-Brunet in (0). Sugeno and Yasukawa underline in (0) how difficult it is to build a fuzzy model when data are scarce and membership functions don't sweep over all the universe of discourse. This may improve the generalisation ability of fuzzy systems (0).

Several methods have been proposed to obtain automatic fuzzy rules from sparse training data.

In (0), the authors first construct fuzzy rules from collect data. Then, they use kernel regressions for generate training data.

Another technique used when classical inference methods produce sparse fuzzy rules is a diffusion procedure based on interpolation to initialize incomplete rules $(0),(0),(0)$.

The objective of this paper is to measure the impact of datum plane covering on the outcome of a fuzzy inference system. Most of optimization methods make the assumption that datum plane is sufficiently covered. If this assumption no longer holds, we will see that these methods cannot work, since it implies that, before optimization, the fuzzy system gives acceptable results. In (0), we analysed the relationship between learning set $\Omega$ and labels of fuzzy inference system. In this paper, we take into account a data density repartition, by the measure of number of available data on intervals of each input variable domain.

All these tecniques take place during optimisation step when our method is used before initialization step. This in turn allows one to isolate unfired rules and to proceed, if necessary, to a partial remodelling of the FIS before training and optimization.

\section{The Tagaki-Sugeno Model}

The model under consideration in this section is a Tagaki-Sugeno model, which corresponds to discretized linear models of order 1 , combined with nonlinear functions.

$y(t)=\sum_{i=1}^{r} h_{i}(z(t))\left(a_{1}^{i} \cdot y(t-i\right.$ decal $)+b_{1}^{i} \cdot u(t-i$ decal $\left.)\right)$

$y(t)$ is the output,

$r$ is the number of linear models,

$z(t)$ a vector which depends lineary or not on the state,

$h_{i}(z(t))>=0, i=1, \cdots, r$ nonlinear functions verifying the convex sum property.

idecal is the time lag between input and its effect, which is specially interesting in our application. 
We have measures about every five minutes and we admit that the effect of insulin (considered in our application)is fast and noticeable ten (idecal=2) minutes later, up to half an hour(idecal =6).

The determination of unknown parameters $a_{1}^{i}$ and $a_{2}^{i}$ is done by the algorithm of recursive least square.

\section{Application to the insulin/glycaemia behavior of diabetics}

\subsection{The available data}

The correlated data "insulin infusion delivery/glycaemia" has been provided by the team of Pr. Pinget, CHU of Strasbourg. They concern the same person and the same insulin. The insulin infusion has been done by an intra-peritoneal route and the glycaemia has been checked by a subcutaneous sensor. Measures of glycaemia have been made every five minutes during 7 days, which corresponds to 1700 measures.

A bolus is a dose of insulin infused manually, in addition to the basic dose, since postprandial glycemia cannot be regulated satisfactorily. The insulin file contains crude data about basic insulin doses as well as boluses. So, a pretraitement of the insulin file has been necessary to produce a file of insulin delivery for the same person every five minutes.

\subsection{Experiments and Validation of the model}

The learning set is composed of the first measures(280 points) that corresponds to insulin infusion and blood glucose concentration of a patient during a day. We take $7(r=7)$ linear models, considering that each model is valid about three and half hours. The mean square error(MSE)is calculated on the totality of the measures(1700 points).

We make experiments by changing the parameter idecal of the model, time lag between an input and its effect.

The test of our modeling method shows that we can predict the glycaemia over a long period (7 days), by considering glycaemia and insulin delivery 15 minute (resp 30-minute) before with an error of about $6 \%($ resp $16 \%$ ), which is a good result compared with current results. However, we see that results obtained are not so good in the last case, when we consider

\begin{tabular}{|c|c|c|}
\hline $\mathrm{r}$ & idecal & MSE \\
\hline 7 & 2 & 0.04 \\
\hline & & \\
\hline 7 & 3 & 0.06 \\
\hline 1 & & \\
\hline 7 & 6 & 0.16 \\
\hline & & \\
\hline 7 & 24 & 1.02 \\
\hline & & \\
\hline
\end{tabular}

Table 1: First Table.

slow effect insulin (with 2-hours delay). In this case, our model has to be refined, by increasing its order.

\section{Datum Plane Covering}

We propose a measure used to pre-validate a fuzzy model. We suppose that there exists a learning set $\Omega=\left\{\left(\mathbf{x}_{j}, d_{j}\right)\right\}$, where $\mathbf{x}_{j}$ is an input vector and $d_{j}$, the corresponding output. We also assume that the desired function $\mathrm{f}$ is defined in

$$
V=\left[a_{1}, b_{1}\right] \times\left[a_{2}, b_{2}\right] \times \ldots \times\left[a_{p}, b_{p}\right]
$$

Usually, to validate a fuzzy inference system, the mean square error (MSE) is calculated on a test set. If the MSE exceeds a threshold, then training is done, using a gradient method. This consists in modifying $C_{j}$ at each presentation of examples from the error $\left(y\left(\mathbf{x}_{j}\right)-d_{j}\right)$.

Unfortunately, in case of model invalidation, we cannot determine never learned rules that cause the gap between the model and the real system. Moreover, if there is an insufficient covering of datum plane, training and finer splitting of input space are inefficient and useless. With the criterion proposed below, we estimate the datum plane coverage and we are able to isolate inactivated rules. Then, partial remodeling of the fuzzy inference system is possible. The study is investigating the relationship between a quantitative variable $X$, number of available data for each input, and a qualitative variable $Y$, labels of membership functions.

When designing a fuzzy system, we attribute to each input $I r$ modalities (or labels) noted $y_{1}, \cdots y_{l}, \cdots y_{r}$. We note $X^{I}$ the variable for the input $I$ of average $\bar{x}^{I}$ and variance $\sigma_{X^{I}}^{2}$. We note $\Omega^{I}$ the corresponding learning set . Each label $y_{l}$ of $I$ defines a subset $\Omega_{l}^{I}$ of $\Omega^{I}$ : we obtain a partition of $\Omega^{I}$ in $m$ classes. We note $n_{l}^{I}=\operatorname{card}\left(\Omega_{l}^{I}\right)$ and $n^{I}=\operatorname{card}\left(\Omega^{I}\right)$. We have $n^{I}=\sum_{l=1}^{m} n_{l}^{I}$. Then, if we consider the restriction de $X^{I}$ to $\left.\Omega_{l}^{I}\right)(l=1, \cdots, m)$, we may define 
the average (noted $\bar{x}_{l}^{I}$ )and the variance (noted $\sigma_{X_{l}^{I}}^{2}$ of $X^{I}$ on this subset:

$$
\begin{gathered}
\bar{x}_{l}^{I}=\frac{\sum_{\omega \in \Omega_{l}^{I}} X(\omega)}{n_{l}^{I}} \\
\sigma_{X_{l}^{I}}^{2}=\frac{\sum_{\omega \in \Omega_{l}^{I}}\left(X(\omega)-\bar{x}_{l}^{I}\right)^{2}}{n_{l}^{I}}
\end{gathered}
$$

We have an index of connection between the datum plane coverage (for an input $I$ ) and the learning set defined by :

$$
s^{I}=\sqrt{\frac{\sigma_{E}^{2}}{\sigma_{X I}^{2}}}
$$

where

$$
\sigma_{X^{I}}^{2}=\sigma_{E}^{2}+\sigma_{R}^{2}
$$

and

$$
\sigma_{E}^{2}=\frac{\sum_{l=1}^{r} n_{l}^{I} *\left(\bar{x}_{l}^{I}-\bar{x}^{I}\right)^{2}}{n^{I}}
$$

and

$$
\sigma_{R}^{2}=\frac{\sum_{l=1}^{r} n_{l}^{I} * \sigma_{X_{l}^{I}}^{2}}{n^{I}}
$$

and

$$
\bar{x}^{I}=\frac{\sum_{l=1}^{r}\left(n_{l}^{I} * \bar{x}_{l}^{I}\right)}{n^{I}}
$$

This index of connection consists in detecting relationships between number of data of the learning set $\Omega^{I}$ and $r_{I}$ labels. This index is low if the features of these labels are not so different (Test 1). When this index is high, it points to that there is a bad repartition of membership functions (Test 3 ). This gives an information about the repartition of data of learning set $\Omega^{I}$ between membership functions.

\subsection{Experiments}

We have made 3 tests for the first input (with two triangular membership functions) using our application. We give in these array the features of these functions. We obtain for the first test a low index(0.035), the second a medium index (0.28) and for the last test a high index (0.84). In the third test, the first membership function is useless and the corresponding rules are inactived. So, we can suppress them without affecting results. We have made a 4th test where three (out of four) membership functions and the associated rules were unnecessary.

\begin{tabular}{|c|c|c|c|}
\hline Test & Center & Left corner & Right corner \\
\hline 1 & 1.4 & 1.6 & 1.0 \\
\hline & & & \\
\hline 2 & 1.0 & 0.6 & 1.0 \\
\hline & & & \\
\hline 3 & 0.2 & 0.2 & 0.2 \\
\hline & & & \\
\hline
\end{tabular}

Table 2: First membership function.

\begin{tabular}{|c|c|c|c|}
\hline Test & Center & Left corner & Right corner \\
\hline 1 & 2.6 & 1.0 & 0.9 \\
\hline & & & \\
\hline 2 & 2.6 & 1.0 & 0.9 \\
\hline & & & \\
\hline 3 & 3.0 & 2.8 & 0.5 \\
\hline & & & \\
\hline
\end{tabular}

Table 3: Second membership function.

\section{2 graphic representation}

We represent sets of data by Box \& Whiskers Plots to underline the relation between number of data and labels of membership functions. The first figure (resp figure 2 and figure 3 ) corresponds to Test1 (resp Test 2 and Test 3 ).

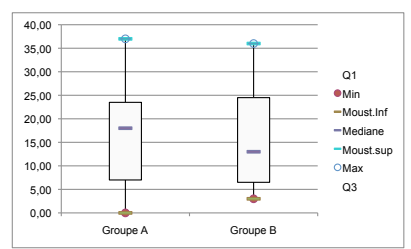

Figure 1

\section{Conclusion}

We have proposed a measure for detecting useless rules and thus pre-validating a fuzzy inference system. When the model is not pre-validated, we have not to carry out next steps, particularly optimization step. 


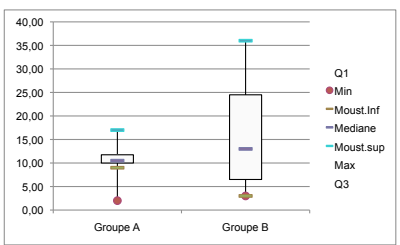

Figure 2

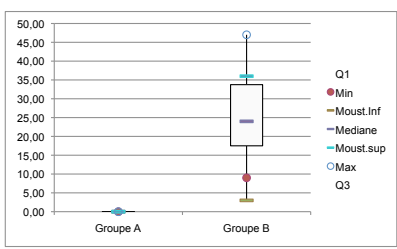

Figure 3

We have shown that this criterion gives useful information about datum plane coverage.

\section{REFERENCES}

Benmakrouha F. Parameter identification in a fuzzy system with insufficient data. Sixth IEEE International Conference on Fuzzy Systems, 1:537 542, July 1997.

F Benmakrouha, C Hespel, E Monnier, An algorithm for rule selection on fuzzy rule-based system applied to the treatment of diabetics and detection fraud in electronic payment, FUZZ-IEEE 2010 Barcelona Spain

P.Y Glorennec, Algorithmes d'apprentissage pour systèmes d'inférence floue Paris France Hermès, 1999.

Sugeno M.,Yasukawa T. "A Fuzzy-Logic-Based Approch to Qualitative Modeling”, IEEE Trans. on Fuzzy Systems, Vol. 1, no 1, February 1993.

Lutaud M. Identification et Contrôle de processus par réseaux Neuro-Flous. Thèse de Doctorat de l'Université d'Evry Val d'Essonne

Baranyi, P. Kczy A general and specialised solid cutting method for fuzzy rule interpolation. in $\mathrm{J}$. BUSEFAL, URA-CNRS, Vol 66, France, 1996, pp 13-22.

A.M. Albisser, B.S. Leibel, T.G. Ewart, et al., An artificial endocrine pancreas , Diabetes, 23 (1974), pp. 389-396.

J.L. Selam, P. Micossi, F.L. Dunn et al., Clinical trial of programmable implantable insulin pump for type I diabetes, Diabetes Care, 15 (1992) pp. 877-885.
G. Bleckert, U.G. Oppel, and E. Salzsieder, Mixed graphical models for simultaneous model identification and control applied to glucose-insulin metabolism ,Comput.Methods Prog.Biomed. 56(1998), no. 2, 141-155.

B. Candas and J. Radziuk, An adaptive plasma glucose controller based on a nonlinear insulin/glucose model, IEEE Trans. Biomed. Eng. 41 (1994), no. 2, 116-123.

Cruz Vega Israel, Wen Yu Multiple fuzzy neural networks modeling with sparse data, Neurocomputing 73 (2010) pp 2446-2453B

Isao Ishibuchi, Takashi Yamamoto Performance Evaluation of Fuzzy Partitions with Different Fuzzification Grades, FUZZ-IEEE'02. Proceedings of the 2002 IEEE International Conference Fuzzy Systems, 2002.

R.S. Parker, F.J. Doyle III, and N.A. Peppas, A modelbased algorithm for blood glucose control in Type I diabetic patients, IEEE Trans. Biomed. Eng. 46 (1999), no. 2, 148-157.

Z. Trajanoski and P. Wach, Fuzzy filter for state estimation of a gluco-regulatory system, Comput. Methods Progr. Biomed. 50 (1996), no. 3, 265273.

T. Johansen Fuzzy model based control: stability,robustness, and performance issues, IEEE Trans.Fuzzy Syst. 2(3)(1994) 221-234

H. Wang, K. Tanaka, M.Griffin, An approach to fuzzy control of nonlinear systems: stability and design issues, IEEE Trans.Fuzzy Syst. 4(1)(1996) 14-23.

S. Cao, N. Rees, G. Feng, Analysis and design for a class of complex control systems., Part I: Fuzzy modelling and identification, Automatica 33(6)(1997) 1017-1028.

J.Gutierrez, F. Fernandez, S. Guadarrama, E. Trillas , "A step towards conceptually improving tagakisugeno's approximation”, IPMU'2002 , III:pp 1789-1794, July 2002.

A. Juditsky, H. Hjalmarsson, A. Benveniste, B. Delyon, L. Ljung, J. Sjberg,and Q. Zhang, Nonlinear black-box models in system identification: mathematical foundations, Automatica J. IFAC 31 (1995), no. 12, 1725-1750.

J. Sjberg, Q. Zhang, L. Ljung, A. Benveniste, B. Delyon, P.-Y. Glorennec, H. Hjalmarsson, and A. Juditsky, Nonlinear black-box modeling insystems identification: a unified overview, Automatica J. IFAC 31 (1995), no. 12, 1691-1724. 